\title{
Double primary hepatic cancer (hepatocellular carcinoma and intrahepatic cholangiocarcinoma) in a single patient: A case report
}

\author{
RONGXING ZHOU ${ }^{1 *}$, MINJIA ZHANG ${ }^{2 *}$, NANSHENG CHENG $^{1}$ and YONG ZHOU ${ }^{1}$ \\ Departments of ${ }^{1}$ Biliary Surgery and ${ }^{2}$ Cardiology, West China Hospital, \\ Sichuan University, Chengdu, Sichuan 610041, P.R. China
}

Received October 24, 2014; Accepted September 4, 2015

DOI: $10.3892 / \mathrm{ol} .2015 .3896$

\begin{abstract}
Double primary hepatic cancer, consisting of hepatocellular carcinoma (HCC) and intrahepatic cholangiocarcinoma (ICC) located separately within a single liver simultaneously, is extremely rare. The present study reports a case of double hepatic nodules, in which HCC and ICC occurred simultaneously in the right hepatic lobe. The 47-year-old male patient, who was a carrier of hepatitis B virus, was admitted to our hospital for physical examination, which revealed two liver masses. The results of initial laboratory tests, including liver function tests, were within normal limits, with the exception of mildly elevated aspartate aminotransferase and alanine aminotransferase, and decreased albumin levels. $\alpha$-fetoprotein was in the normal range, while carbohydrate antigen 19-9 was marginally elevated. Abdominal ultrasonography and enhanced computed tomography revealed two tumors located in segments (S) VI and VII of the liver, respectively, with malignant behavior. Examination of the two masses following resection of S VI and VII confirmed a diagnosis of combined HCC and ICC. After 8 months of follow-up, no signs of recurrence have been observed with chemical therapy.
\end{abstract}

\section{Introduction}

Primary liver cancer (PLC) ranks as the 5th most common cancer worldwide and the 3rd most prevalent in China $(1,2)$. The majority of PLCs are hepatocellular carcinoma (HCC) or intrahepatic cholangiocarcinoma (ICC); however, combined $\mathrm{HCC}$ and cholangiocarcinoma (CC) in a single liver (cHCC-CC) is reported to account for $0.4-14.2 \%$ of cases (3-7). Allen and Lisa (8) classified cHCC-CC into three subtypes: Type A,

Correspondence to: Professor Yong Zhou, Department of Biliary Surgery, West China Hospital, Sichuan University, 37 Guoxue Alley, Chengdu, Sichuan 610041, P.R. China

E-mail: zhourongxing@163.com

\section{*Contributed equally}

Key words: diagnosis, mass, intrahepatic cholangiocarcinoma, hepatocellular carcinoma, treatment separate nodules of $\mathrm{HCC}$ and $\mathrm{CC}$; type $\mathrm{B}$, contiguous masses of $\mathrm{HCC}$ and $\mathrm{CC}$; and type $\mathrm{C}$, individual masses intermingling, with components of $\mathrm{HCC}$ and $\mathrm{CC}$. Type $\mathrm{C}$ is regarded to be the real $\mathrm{cHCC}-\mathrm{CC}$ classification, and the majority of cases of cHCC-CC reported are type C (9). Types A or B, also known as double separate masses of HCC and ICC, are extremely rare in clinical practice, particularly type A. It has been reported that clinicopathological characteristics and surgical outcomes generally differ between types A, B and C, although this is poorly understood at present (10). The current study reports the case of a patient diagnosed with HCC and ICC occurring as two separate nodules.

\section{Case report}

A 47-year-old male was admitted to West China Hospital of Sichuan University (Chengdu, China) in February 2014 without any symptoms and tumor was discovered by physical examination.. Physical examination revealed two nodules in the right lobe of the patient's liver. No history of drug or alcohol abuse was reported, however, the patient was positive for hepatitis B virus (HBV). Abdominal ultrasound, enhanced computed tomography (CT) and initial laboratory tests were scheduled. The laboratory test results revealed that the blood hemoglobin (HGB) levels and white blood cell and platelet counts were all within the normal range. However, a liver function test revealed that aspartate aminotransferase and alanine aminotransferase levels were mildly elevated, and albumin levels were found to be decreased, with a normal total bilirubin concentration. A HBV DNA titer of $5 \times 10^{3}$ copies/ 1 was detected. Levels of the tumor markers $\alpha$-fetoprotein (AFP; $2.1 \mu \mathrm{g} / \mu \mathrm{l}$; normal range, $0-8 \mu \mathrm{g} / \mu \mathrm{l}$ ), carcinoembryonic antigen $(2.1 \mu \mathrm{g} / \mathrm{ml}$; normal range, $0-5 \mu \mathrm{g} / \mathrm{ml})$ and carbohydrate antigen (CA) 125 (16.2 U/ml; normal range, 0-35 U/ml) were all within the normal limits, however, the CA19-9 level was marginally elevated. The abdominal ultrasound revealed two tumors in segment (S) VII (tumor A) and S VI (tumor B), with liver echo enhancement. In addition, enhanced spiral CT scanning of the upper abdomen showed that the two masses, tumors A and B, were both located in the right posterior lobe and measured $4 \mathrm{~cm}$ and $1 \mathrm{~cm}$ in diameter, respectively (Fig. 1). Esophageal varices were also observed. Enhanced CT imaging revealed that tumor A was heterogeneously enhanced in the arterial phase, and enhancement decreased in the portal venous phase, 
A

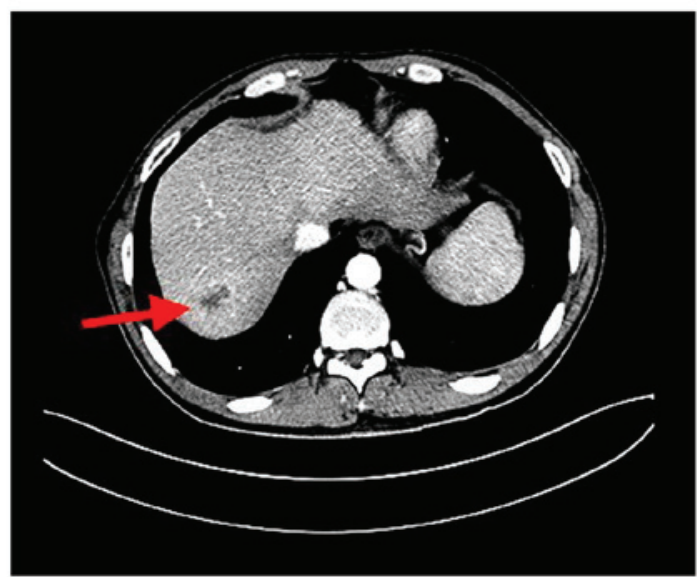

B

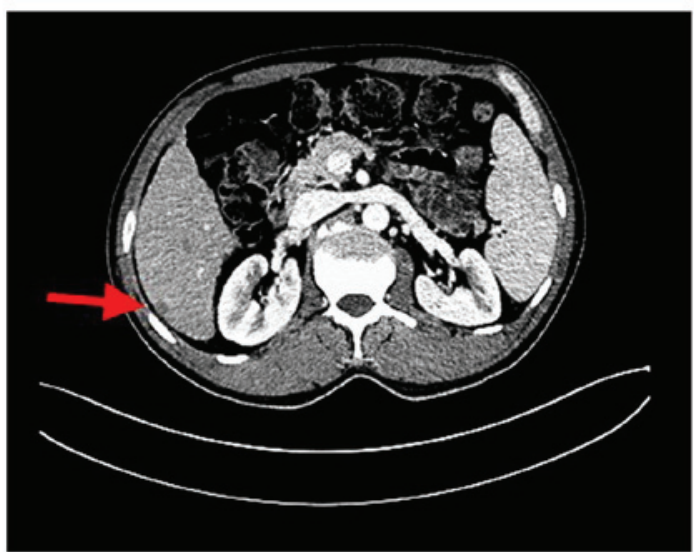

Figure 1. Computed tomography scan of the liver in the venous phase. Red arrows indicate (A) tumor A (hepatocellular carcinoma) in segment VII and (B) tumor B (intrahepatic cholangiocarcinoma) in segment VI.

A

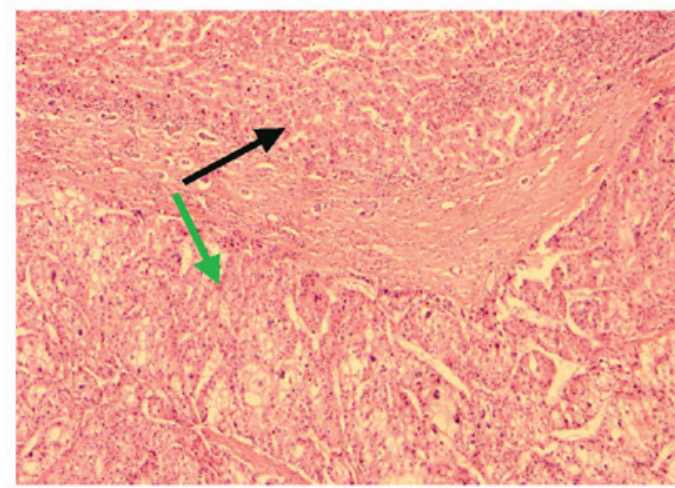

B

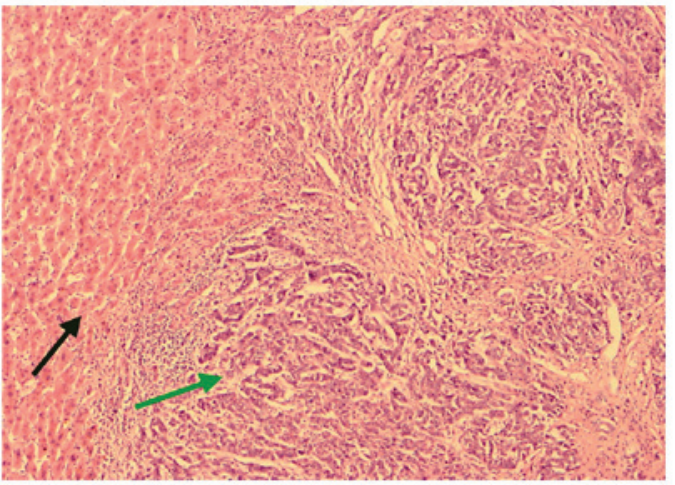

Figure 2. Light microscopy of (A) tumor A (hepatocellular carcinoma) and (B) tumor B (intrahepatic cholangiocarcinoma). Black arrows indicate normal liver tissue, while green arrows indicate carcinoma tissue (hematoxylin and eosin staining; magnification, $\mathrm{x} 100$ ).
A

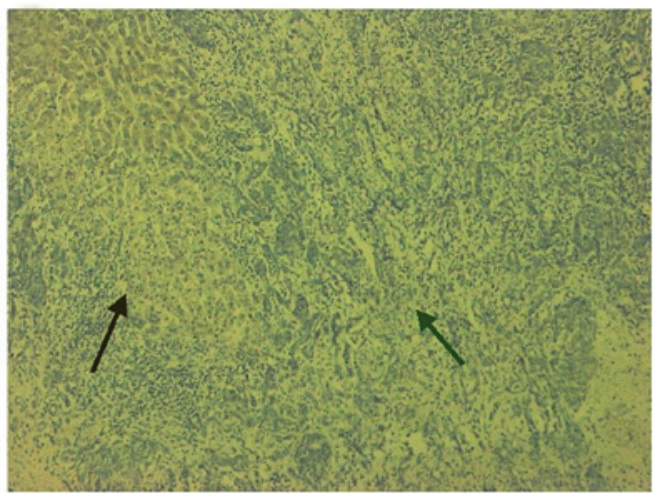

B

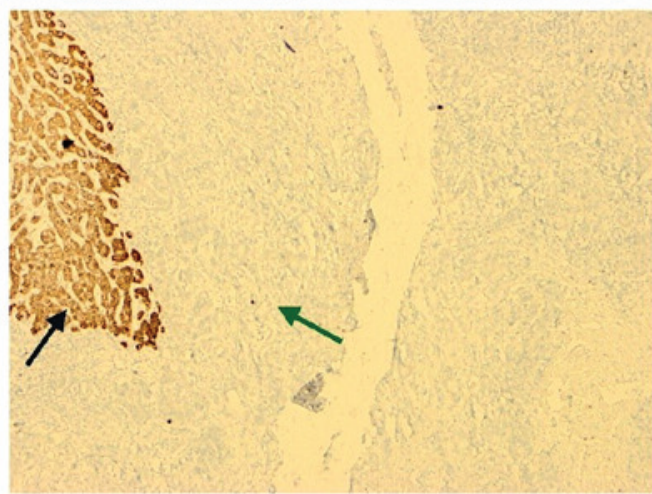

C

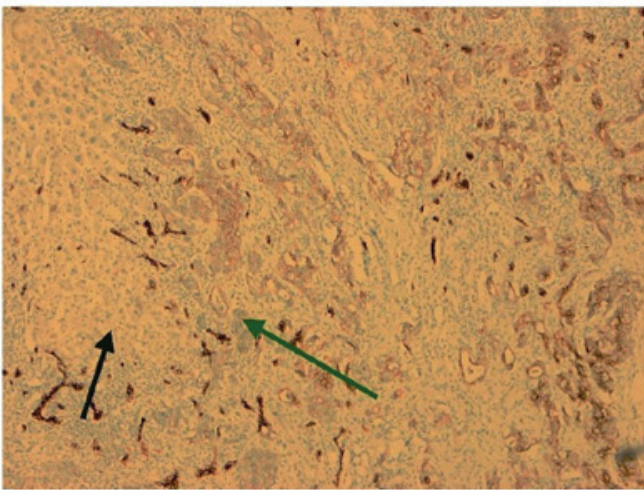

D

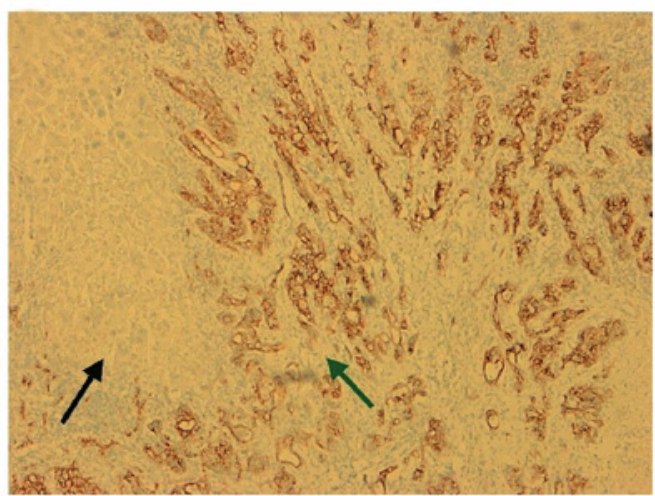

Figure 3. Immunohistochemical analysis of tumor B (intrahepatic cholangiocarcinoma). Black arrows indicate normal liver tissue, and green arrows indicate adenocarcinoma tissue. Figures show negative staining of adenocarcinoma tissue for (A) $\alpha$-fetoprotein and (B) hepatocyte antigen, and positive staining for (C) CK7 and (D) CK19 (magnification, x100). CK, cytokeratin.

while tumor B was heterogeneously enhanced in these two phases.

Based on these findings, primary HCC with intrahepatic metastasis was the initial diagnosis; there was no evidence of extrahepatic lesions on enhanced brain and chest CT imaging 
and bone scanning. Therefore, surgical resections of liver S VI and VII were performed under full anesthesia within $110 \mathrm{~min}$. The patient experienced $200 \mathrm{ml}$ blood loss during surgery, and no blood transfusion was required. Following an uneventful recovery, the patient was discharged on the 7 th day after surgery. The biopsy of the liver revealed cirrhosis with an Ishak score of 6 (11). Tumor A was found to be moderately differentiated HCC with a complete capsule and without tumor thrombus. By contrast, tumor B was a poorly differentiated adenocarcinoma with liver capsule violation (Fig. 2). In order to elucidate the source of tumor $\mathrm{B}$, an immunohistochemical analysis of the tumor was performed. The cells of tumor B stained positively for cytokeratins (CKs) 7 and 19, and negatively for hepatocyte antigen (Hepa) and AFP (Fig. 3). Thus, these finding indicated that tumor B had a biliary origin, confirming a diagnosis of ICC. During the 8 months of follow-up, the patient has undergone liver ultrasonography and tumor marker tests at 3-month intervals, and no signs of recurrence have been observed. No further treatment has been administered.

The patient provided written informed consent for the publication of this report.

\section{Discussion}

The simultaneous occurrence of HCC and ICC in a single liver, in the form of separated nodules, is extremely rare. To the best of our knowledge, there have been no previous reports about the incidence of the type A cHCC-CC, and only several English language case reports are available in the literature (12).

It is well-established that HCC is closely associated with $\mathrm{HBV}$ or hepatitis $\mathrm{C}$ virus (HCV) infections $(2,13)$, while HBV or HCV infections are also risk factors for ICC (14). In the present report, the patient had a long history of HBV infection, including increasing HBV DNA titers, and no standard antiviral treatments were administered.

Tumor markers are intrinsically linked to certain tumors, however, tumor markers cannot necessarily be used to confirm an exact final diagnosis (15). For example, the level of blood CA19-9, a tumor marker of ICC, may also increase when HCC invades the biliary tract; therefore, elevated levels cannot conclusively diagnose a liver mass as ICC, but may suggest the possibility of ICC. In the present report, the AFP level of the patient, which is regarded to be the most significant tumor maker of HCC (16), was within the normal limits, however, CA19-9 was marginally elevated. Thus, whilst they provide some information, tumor markers cannot be used as the sole foundation of the diagnosis, and the final diagnosis depends on pathological assessment of biopsied specimens.

The pathological characteristics of HCC and ICC also differ from one another: According to the consensus of pathological immunohistochemical examinations, Hepa and AFP are reliable markers for HCC (17), while CK7 and CK19 are valuable markers for differentiating ICC from HCC (18). In the present report, tumor A was a typical HCC mass, and tumor B cells stained positively for CK7, CK19 and CK20, and negatively for Hepa and AFP. Negative AFP and Hepa staining indicated that the tumor did not originate from hepatocytes, while the CK7 and CK19 positive staining suggested that the tumor arose from cholangiocytes $(19,20)$.
The use of abdominal ultrasound to detect liver masses is common for screening and follow-up; however, the characterization of a malignant tumor by ultrasound often leads to ambiguity due to the variable and non-specific features observed using this modality, and is limited to the experience of the sonologist (21). HCC and ICC masses may perform differently on enhanced CT imaging: ICC typically appears with peripheral enhancement on the early phase, and mild centripetal progression of enhancement in the venous phase, while HCC generally exhibits enhancement in the whole tumor during the hepatic arterial phase and negative enhancement during the portal venous phase ('fast in and out') (22). The biomolecular mechanism of enhancement differences on spiral CT between HCC and ICC is related to the differential expression of vascular endothelial growth factor (23). However, it remains difficult to characterize tumors of small diameter or with atypical features by CT imaging. In the present report, the diameter of the ICC nodule in S VI was $\sim 1 \mathrm{~cm}$, and the CT image revealed irregular enhancement in the arterial and venous phases; thus, it could not be fully distinguished from certain metastatic tumors (24). Therefore, even combining the tumor marker and imaging findings, correctly diagnosing the double primary hepatic masses as HCC and ICC preoperatively was still challenging.

Hepatectomy is the primary treatment option for all malignant liver tumors, however, different approaches to lymph node dissection are usually taken between HCC and ICC (25). It is essential to dissect lymph nodes for ICC, but not for HCC (26). In the present case, the patient only underwent liver resection and not lymph node dissection, due to the misdiagnosis of tumor B preoperatively. Despite this, the patient has experienced disease-free survival to date, owing to the small diameter of the tumor and absence of lymph node metastasis. It may be possible to avoid uncertainty regarding the necessity for lymph node dissection by implementing frozen section biopsy intraoperatively, when the evidence from preoperative tests is unable to fully eliminate the possibility of ICC (10).

In summary, the present study reported an extremely rare case of PLC consisting of double hepatic tumors, with HCC and ICC occurring simultaneous at separate locations within a single liver. In such cases, it is difficult to determine a diagnosis preoperatively due to uncharacteristic performances on tumor marker analysis and imaging studies; the final diagnosis depends on pathological and immunohistochemical examination of biopsy specimens. Surgery remains the primary treatment option. The present case highlights the potential value of frozen section biopsy performed intraoperatively that may alert surgeons to the necessity for lymph node dissection.

\section{References}

1. Parkin DM, Bray F, Ferlay J and Pisani P: Estimating the world cancer burden: Globocan 2000. Int J Cancer 94: 153-156, 2001.

2. Chan AC, Cheung TT, Fan ST, Chok KS, Chan SC, Poon RT and Lo CM: Survival analysis of high-intensity focused ultrasound therapy versus radiofrequency ablation in the treatment of recurrent hepatocellular carcinoma. Ann Surg 257: 686-692, 2013

3. Jarnagin WR, Weber S, Tickoo SK, Koea JB, Obiek we S, Fong Y, DeMatteo RP, Blumgart LH and Klimstra D: Combined hepatocellular and cholangio-carcinoma: Demographic, clinical and prognostic factors. Cancer 94: 2040-2046, 2002. 
4. Tang D, Nagano H, Nakamura M, Wada H, Marubashi S, Miyamoto A, Takeda Y, Umeshita K, Dono K and Monden M: Clinical and pathological eatures of Allen's type C classification of resected combined hepatocellular and cholangiocarcinoma: A comparative study with hepatocellular carcinoma and cholangiocellular carcinoma. J Gastrointest Surg 10: 987-998, 2006.

5. Goodman ZD, Ishak KG, Langloss JM, Sesterhenn IA and Rabin L: Combined hepatocellular-cholangiocarcinoma. A histologic and immunohistochemical study. Cancer 55: 124-135, 1985.

6. Kassahun WT and Hauss J: Management of combined hepatocellular and cholangiocarcinoma. Int J Clin Pract 62: 1271-1278, 2008.

7. Kim KH, Lee SG, Park EH, et al. Surgical treatments and prognoses of patients with combined hepatocellular carcinoma and cholangiocarcinoma. Ann Surg Oncol. 2009;16(3):623-629.

8. Allen RA and Lisa JR: Combined liver cell and bile duct carcinoma. Am J Pathol 25: 647-655, 1949.

9. Yano Y, Yamamoto J, Kosuge T, Sakamoto Y, Yamasaki S, Shimada K, Ojima H, Sakamoto M, Takayama $T$ and Makuuchi M: Combined hepatocellular and cholangiocarcinoma: a clinicopathologic study of 26 resected cases. Jpn J Clin Oncol 33: 283-287, 2003.

10. Cao J, Huang L, Liu C, Li J, Zhang X, Shen J, Li J, Lu L, Xu F, Yan J, Wu M, Lau WY and Yan Y: Double primary hepatic cancer hepatocellular carcinoma and intrahepatic cholangiocarcinoma) in a single patient: A clinicopathologic study of 35 resected cases. J Gastroenterol Hepatol 28: 1025-1031, 2013.

11. Everhart JE, Wright EC, Goodman ZD, Dienstag JL, Hoefs JC, Kleiner DE, Ghany MG, Mills AS, Nash SR, Govindarajan S, et al; HALT-C Trial Group: Prognostic value of Ishak fibrosis stage: Findings from the hepatitis $\mathrm{C}$ antiviral long-term treatment against cirrhosis trial. Hepatology 51: 585-594, 2010.

12. Maganty K, Levi D, Moon J, Bejarano PA, Arosemena L, Tzakis A and Martin P: Combined hepatocellular carcinoma and intrahepatic cholangiocarcinoma: Outcome after liver transplantation. Dig Dis Sci 55: 3597-3601, 2010.

13. Franssen B, Alshebeeb K, Tabrizian P, Marti J, Pierobon ES, Lubezky N, Roayaie S, Florman S and Schwartz ME: Differences in surgical outcomes between hepatitis B- and hepatitis C-related hepatocellular carcinoma: A retrospective analysis of a single North American center. Ann Surg 260: 650-656, 2014.

14. Shaib YH, El-Serag HB, Davila JA, Morgan R and McGlynn KA: Risk factors of intrahepatic cholangiocarcinoma in the United States: A case-control study. Gastroenterology 128: 620-626, 2005

15. Mant D and Primrose J: CEA monitoring in colorectal cancer is not a waste of time. BMJ 18: 348, 2014
16. Shim JH, Yoon DL, Han S, Lee YJ, Lee SG, Kim KM, Lim YS, Lee HC, Chung YH and Lee YS: Is serum alpha-fetoprotein useful for predicting recurrence and mortality specific to hepatocellular carcinoma after hepatectomy? A test based on propensity scores and competing risks analysis. Ann Surg Oncol 19: 3687-3696, 2012.

17. Jeng KS, Sheen IS, Jeng WJ, Yu MC, Hsiau HI, Chang FY and Tsai HH: Activation of the sonic hedgehog signaling pathway occurs in the CD133 positive cells of mouse liver cancer Hepa 1-6 cells. Onco Targets Ther 6: 1047-1055, 2013.

18. Massani M, Stecca T, Fabris L, Caratozzolo E, Ruffolo C, Furlanetto A, Morton S, Cadamuro M, Strazzabosco M and Bassi N: Isolation and characterization of biliary epithelial and stromal cells from resected human cholangiocarcinoma: A novel in vitro model to study tumor-stroma interactions. Oncol Rep 30: 1143-1148, 2013

19. Moritoki Y, Ueno Y, Kanno N, Yamagiwa Y, Fukushima K, Gershwin ME and Shimosegawa T: Amniotic epithelial cell-derived cholangiocytes in experimental cholestatic ductal hyperplasia. Hepatol Res 37: 286-294, 2007.

20. Xu J, Sasaki M, Harada K, Sato Y, Ikeda H, Kim JH, Yu E and Nakanuma Y: Intrahepatic cholangiocarcinoma arising in chronic advanced liver disease and the cholangiocarcinomatous component of hepatocellular cholangiocarcinoma share common phenotypes and cholangiocarcinogenesis. Histopathol 59: 1090-1099, 2011.

21. Stuntz R, Kochert E, Kehrl T and Schrading W: The effect of sonologist experience on the ability to determine endotracheal tube location using transtracheal ultrasound. Am J Emerg Med 32: 267-269, 2014.

22. Kim TK, Jang HJ and Wilson SR: Imaging diagnosis of hepatocellular carcinoma with differentiation from other pathology. Clin Liver Dis 9: 253-279, 2005.

23. Wagner K, Peters M, Scholz A, Benckert C, Ruderisch HS, Wiedenmann B and Rosewicz S: Activin A stimulates vascular endothelial growth factor gene transcription in human hepatocellular carcinoma cells. Gastroenterology 126: 1828-1843, 2004.

24. Kang Y, Lee JM, Kim SH, Han JK and Choi BI: Intrahepatic mass-forming cholangiocarcinoma: Enhancement patterns on gadoxetic acid-enhanced MR images. Radiology 264: 751-760, 2012.

25. Amini N, Ejaz A, Spolverato G, Maithel SK, Kim Y and Pawlik TM: Management of lymph nodes during resection of hepatocellular carcinoma and intrahepatic cholangiocarcinoma: A systematic review. J Gastrointest Surg 18: 2136-2148, 2014.

26. Popescu I and Dumitrascu T: Curative-intent surgery for hilar cholangiocarcinoma: Prognostic factors for clinical decision making. Langenbecks Arch Surg 399: 693-705, 2014. 